\title{
TAXA DE DECOMPOSIÇÃO DA SERAPILHEIRA E ATIVIDADE MICROBIANA EM ÁREA DE CAATINGA
}

\author{
Patrícia Carneiro Souto ${ }^{1}$, Jacob Silva Soutoํㅡ, Rivaldo Vital dos Santos ${ }^{1}$, Ivonete Alves Bakke ${ }^{1}$, \\ Francisco das Chagas Vieira Sales ${ }^{2 *}$, Bruna Vieira de Souza ${ }^{3}$
}

*Autor para correspondência: franciscoef@yahoo.com.br

\begin{abstract}
RESUMO: Objetivando avaliar a decomposição da serapilheira e a atividade microbiana em área de Caatinga preservada, conduziuse um experimento na Reserva Particular do Patrimônio Natural Fazenda Tamanduá, em Santa Terezinha, Estado da Paraíba. A taxa de decomposição foi determinada com o uso de sacolas de náilon, contendo $30 \mathrm{~g}$ de serapilheira, que foram dispostas na superfície do solo em setembro de 2003, sendo retiradas 20 sacolas mensalmente até setembro de 2005 . O material coletado foi seco em estufa e pesado para avaliar a perda de peso em relação ao peso inicial. A atividade microbiana foi estimada mensalmente pela quantificação do dióxido de carbono $\left(\mathrm{CO}_{2}\right)$ liberado no processo de respiração edáfica, a partir da superfície do solo, e capturado por solução de $\mathrm{KOH}$. A perda de peso da serapilheira, após um ano, foi de 41,19\% e, após dois anos, foi de 48,37\%, evidenciando uma decomposição mais acelerada no primeiro ano. A análise dos dados mostrou a influência da estação do ano na decomposição de serapilheira e da temperatura na atividade microbiana.
\end{abstract}

Palavras-chave: Resíduos florestais, respiração edáfica, floresta seca.

\section{RATE OF LITTER DECOMPOSITION AND MICROBIAL ACTIVITY IN AN AREA OF CAATINGA}

\begin{abstract}
In order to evaluate the decomposition of litter and microbial activity in an area of preserved Caatinga, an experiment was conducted in the Natural Heritage Private Reserve Tamanduá Farm in Santa Terezinha county, State of Paraiba. The decomposition rate was determined by using litter bags containing $30 \mathrm{~g}$ of litter, which were arranged on the soil surface in September 2003 and 20 bags were taken each month until September 2005. The collected material was oven dried and weighed to assess weight loss compared to initial weight. Microbial activity was estimated monthly by the quantification of carbon dioxide (CO) released into the edaphic breathing process from the soil surface, and captured by KOH solution. Weight loss of litter after one year was $41.19 \%$ and, after two years, was 48.37\%, indicating a faster decomposition in the first year. Data analysis showed the influence of season on litter decomposition and temperature on microbial activity.
\end{abstract}

Key words: Forest residues, edaphic breathing, dry forest.

\section{INTRODUÇÃO}

A Caatinga apresenta variada cobertura vegetal, determinadas em grande parte, pelo clima, relevo e embasamento geológico (RODAL et al., 2008). A caducidade natural de material vegetal forma uma camada de serapilheira que protege o solo na estação seca e, logo nas primeiras chuvas é iniciada a incorporação ao solo por ação dos microrganismos decompositores, configurando o principal processo de ciclagem de nutrientes.

A serapilheira sofre, inicialmente, decomposição parcial da mesofauna concomitantemente à ação decompositora dos microrganismos. Parte do carbono presente nos resíduos é liberada para a atmosfera como $\mathrm{CO}_{2}$ e o restante passa a fazer parte da matéria orgânica do solo (BAYER; MIELNICZUK, 1999).

Koukoura et al. (2003) afirmam que as espécies de plantas em ambientes semiáridos podem diferir quanto às taxas de decomposição por causa de variações interespecíficas na qualidade da serapilheira em particular.

Os métodos que têm sido bem utilizados para estimar a decomposição da serapilheira são através da respiração do solo (determina a produção de $\mathrm{CO}_{2}$ pela atividade biológica do solo), o valor $\mathrm{K}$, (que é a relação entre a quantidade de material que cai do dossel e a que está depositada sobre o solo), e avaliações diretas por meio de medidas da perda de massa em sacolas de náilon (ANDRADE, 1997).

Para Savin et al. (2001), a respiração edáfica é um método comum e simples de avaliar a atividade biológica no solo. Vanhala (2002) relata que esse é um método que permite estimar a atividade microbiana total, sendo de fácil execução e baixos custos. A taxa de respiração edáfica, medida no campo, reflete a emissão real do $\mathrm{CO}_{2}$ no solo florestal, estimando, por exemplo, o estoque de carbono do ecossistema.

\footnotetext{
${ }^{1}$ Universidade Federal de Campina Grande - Patos, Paraíba, Brasil

${ }^{2}$ Universidade Federal Rural de Pernambuco - Recife, Pernambuco, Brasil

${ }^{3}$ Fundação para o Desenvolvimento Sustentável do Araripe - Crato, Ceará, Brasil
}

Cerne, Lavras, v. 19, n. 4, p. 559-565, out./dez. 2013 
A velocidade de produção de $\mathrm{CO}_{2}$ tem sido utilizada para compreender melhor as funções da população decompositora e a relação com as condições ambientais (ASSIS JUNIOR et al., 2003; SOUTO et al., 2002). A degradação de diferentes resíduos depende das condições locais e regionais (clima, solo, vegetação, fauna) e microrganismos decompositores (TAUK, 1990).

Embora tenha grande número de trabalhos sobre os processos de decomposição nos mais variados biomas (GAMA-RODRIGUES et al., 2003; HOFMAN et al., 2004), verifica-se escassez de informação sobre a decomposição e a ciclagem de nutrientes em área de Caatinga, com destaque para os trabalhos de Souto et al. (2002, 2004).

Diante da importância do processo de decomposição do material orgânico para a sustentabilidade do ecossistema, neste estudo, objetivou-se quantificar a velocidade de decomposição da serapilheira e a atividade microbiana nos períodos diurno e noturno em área de Caatinga.

\section{MATERIAL E MÉTODOS}

O trabalho foi realizado na Reserva Particular do Patrimônio Natural (RPPN) situada na Fazenda Tamanduá, localizada no município de Santa Terezinha (PB). Segundo a classificação climática de Köppen, o clima da região é o BSh semiárido, com precipitação média anual de $600 \mathrm{~mm}$, com dados pluviométricos coletados na propriedade. $\mathrm{Na}$ RPPN foram demarcados sete transectos, com distância de $200 \mathrm{~m}$ entre si.

O experimento teve a duração de dois anos (Período 1: outubro de 2003 a setembro de 2004 e Período 2: outubro de 2004 a setembro de 2005). Para avaliar a decomposição da serapilheira, em agosto de 2003 foi coletada na área de estudo a serapilheira acumulada no solo, sendo encaminhada ao Laboratório de Nutrição Mineral de Plantas/ UFCG em Patos (PB) para secagem em estufa $\left( \pm 65^{\circ} \mathrm{C}\right)$ até peso constante. Em seguida, a serapilheira foi separada em porções de $30,0 \mathrm{~g}$ e acondicionadas em sacolas de náilon medindo $20,0 \mathrm{~cm} \mathrm{x}$ $20,0 \mathrm{~cm}$, com malha de $1,0 \mathrm{~mm}^{2}$ (MANSON, 1980), em um total de 480 sacolas.

Em setembro de 2003, foram distribuídas em cada transecto 24 sacolas de náilon, fixadas na superfície do solo por piquetes. Mensalmente, foi recolhida, aleatoriamente, uma sacola por transecto e, no laboratório, o material vegetal foi cuidadosamente retirado das sacolas de náilon e limpos para retirada do solo aderido. Após esses procedimentos, foram acondicionados em sacos de papel devidamente identificados e encaminhados para secagem em estufa $\mathrm{a} \pm 65^{\circ} \mathrm{C}$ até peso constante. A massa residual foi determinada em balança de precisão de $0,01 \mathrm{~g}$, estimandose a velocidade de decomposição ( $\mathrm{g}$ mês ${ }^{-1}$ ) em relação ao peso inicial.

Para calcular a taxa de decomposição $(\mathrm{k})$ foi utilizada a equação: $C=C_{o} e^{-k t}$, onde: $\mathrm{C}$ é a massa final das amostras; $\mathrm{C}_{0}$ é a massa inicial (30 g); t, o tempo decorrido na experimentação (730 dias) e $\mathrm{k}$ a constante de decomposição (PARDO et al., 1997). Para estimar o período de meia vida ou período necessário para que $50 \%$ da biomassa fossem transformadas, utilizou-se a equação: $t_{0,5}=\ln \frac{2}{k}$ (COSTA; ATAPATTU, 2001).

A atividade microbiana, medida pela absorção do $\mathrm{CO}_{2}$, segundo a metodologia descrita por Grisi (1978), foi determinada mensalmente nos transectos 1,2 e 3 . Foram utilizados 20 recipientes de vidro, cada um contendo $10 \mathrm{~mL}$ de solução $0,5 \mathrm{~N}$ de $\mathrm{KOH}$, sendo deixado em cada transecto três recipientes com solução, totalizando nove recipientes no período diurno ( $07 \mathrm{~h}$ às $19 \mathrm{~h})$ e noturno de ( $19 \mathrm{~h}$ às $07 \mathrm{~h}$ ) e duas provas para cada período.

Os recipientes, depois de destampados, foram cobertos imediatamente com baldes de PVC com $26 \mathrm{~cm}$ de altura e $23,5 \mathrm{~cm}$ de diâmetro, cobrindo uma área do solo sem vegetação de $433,74 \mathrm{~cm}^{2}$, sendo as bordas dos baldes enterradas cerca de $3,0 \mathrm{~cm}$ no solo. Após 12 horas, correspondentes ao período diurno ou noturno, os recipientes foram recolhidos e levados ao Laboratório para titulação. A quantificação do $\mathrm{CO}_{2}$ desprendido do solo foi feita por meio da titulação com solução padrão ácida $(\mathrm{HCl}$ a $0,1 \mathrm{~N})$, após a precipitação do íon carbonato presente nas amostras, inclusive nas amostras controle. A massa de $\mathrm{CO}_{2}$ desprendida por unidade de área e tempo foi obtida considerando a massa total desprendida no período de permanência na área e a área do balde, utilizando-se a seguinte equação:

$m_{\mathrm{CO}_{2}}=\frac{352 \times\left(\Delta V_{A}-\Delta V_{C}\right) \times N_{B} \times N_{A}}{3 \times P \times A_{B}} \times 10^{4}$,

onde: $\mathrm{m}_{\mathrm{CO}_{2}}=$ massa de $\mathrm{CO}_{2}$ em mg. $\mathrm{m}^{-2} \cdot \mathrm{h}^{-1} ; \Delta \mathrm{V}_{\mathrm{A}}=$ diferença de volume de $\mathrm{HCl}$ gasto na primeira e segunda etapa da titulação da amostra $(\mathrm{mL}) ; \Delta \mathrm{V}_{\mathrm{C}}=$ diferença de volume de $\mathrm{HCl}$ gasto na primeira e segunda etapa da titulação do controle $(\mathrm{mL}) ; \mathrm{N}_{\mathrm{A}}=$ concentração de $\mathrm{HCl}$, em n-eq L ${ }^{-1}$; $\mathrm{N}_{\mathrm{B}}=$ concentração de $\mathrm{KOH}$, em n-eq $\mathrm{L}^{-1} ; \mathrm{P}=$ período de permanência da amostra no solo (horas); $A_{B}=$ área de abrangência do balde $\left(\mathrm{cm}^{2}\right)$. 
Mensalmente, a temperatura do solo foi medida na superfície e a $15,0 \mathrm{~cm}$ de profundidade com auxílio de termômetro digital, correspondendo aos turnos diurno e noturno. Concomitantemente, foi determinado o conteúdo de água no solo, em amostras coletadas a $15,0 \mathrm{~cm}$ de profundidade, segundo a metodologia da Empresa Brasileira de Pesquisa Agropecuária - EMBRAPA (1997).

O delineamento experimental para a análise da decomposição da serapilheira foi em blocos casualizados com 20 repetições, com os tratamentos em arranjo fatorial 12 x 2 (meses x ano). Para a estimativa da atividade microbiana, foi utilizado o delineamento experimental em blocos casualizados com 3 repetições, com os tratamentos em arranjo fatorial $2 \times 12 \times 2$ (períodos x meses x ano).

Os dados obtidos na avaliação da decomposição e da respiração edáfica foram submetidos à Análise de Variância. Havendo diferenças significativas no teste F da análise de variância, fez-se o teste de médias pelo método de Tukey a 5\%. Para as análises estatísticas foi utilizado o Programa Statistica Visual GLM.

\section{RESULTADOS E DISCUSSÃO}

Na Figura 1, encontram-se as médias mensais da pluviosidade e o conteúdo de água no solo. A precipitação total para o Período 1 (outubro de 2003 a setembro de 2004) foi de $830,8 \mathrm{~mm}$, valor superior ao obtido para o Período 2 (outubro de 2004 a setembro de 2005), que foi de $658,7 \mathrm{~mm}$.

Quanto ao conteúdo de água no solo, observouse que os valores acima de $10 \%$ no Período 1, foram registrados nos meses de janeiro e abril com 19,7 e 12,5\%, respectivamente, e, no Período 2, apenas no mês de março com $15,7 \%$. Nos meses de maiores precipitações, houve aumento no conteúdo de água no solo, com decréscimos imediatos quando havia diminuição no regime hídrico. Portanto, o regime pluviométrico na região apresenta alta variabilidade espacial e temporal, sendo de fundamental importância o monitoramento das condições atmosféricas e conteúdo de água no solo na avaliação do processo de decomposição e atividade dos microrganismos edáficos.

Observa-se, na Figura 2, a ocorrência de temperaturas acima de $30^{\circ} \mathrm{C}$, na superfície e a $15 \mathrm{~cm}$ de profundidade, nos meses de outubro, novembro de 2003 e agosto, setembro de 2004 do Período 1, e nos meses de outubro, novembro, dezembro de 2004 e agosto, setembro de 2005 do Período 2. Nesses meses, houve pluviosidade apenas em dezembro de 2004. Nos meses em que ocorreram precipitações, nos dois períodos, ocorreram decréscimos de quase $10^{\circ} \mathrm{C}$, ficando a temperatura abaixo dos $30^{\circ} \mathrm{C}$ nos meses de janeiro a julho de 2003, e de março a julho de 2005.

Na Figura 3, observa-se a curva de decomposição da serapilheira contida nas sacolas de náilon, distribuídas nos transectos e expostas às condições climáticas naturais, no período de outubro de 2003 a setembro de 2005.

Nos três primeiros meses, o percentual remanescente ficou acima dos $90 \%$. Como o experimento foi instalado na estação seca, os baixos valores de pluviosidade (Figura 1) e a temperatura do solo elevada (Figura 2), tornaram o processo de decomposição mais lento, indicando a influência climática na atividade dos organismos decompositores.

Em trabalho clássico, Witkamp (1966) salienta que as altas temperaturas favorecem as atividades biológicas, no entanto, aumentam o dessecamento do solo e do material. Como as espécies da Caatinga são heliófitas, a maioria das folhas apresenta textura rígida e, por isso, demoram mais a umedecer, o que dificulta a ação dos organismos nos períodos mais secos, tornando o processo de decomposição mais lento.

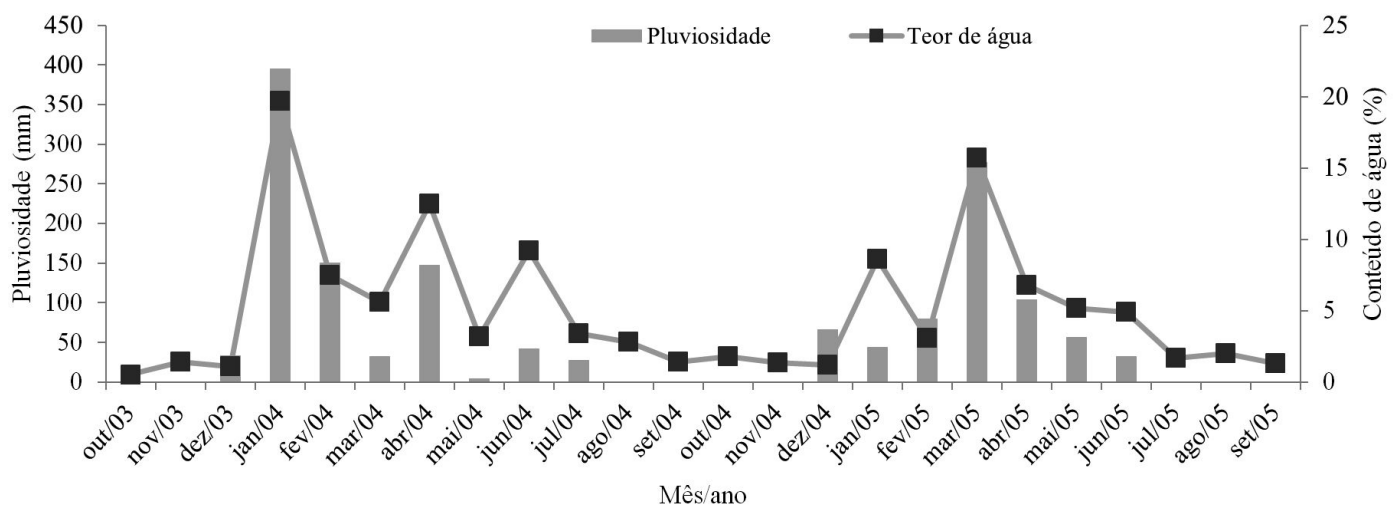

Figura 1 - Pluviosidade (mm) e conteúdo de água no solo (\%) durante o período experimental, em Santa Terezinha, Paraíba.

Figure 1 - Rainfall ( $\mathrm{mm}$ ) and soil water content (\%) during the experimental period.

Cerne, Lavras, v. 19, n. 4, p. 559-565, out./dez. 2013 


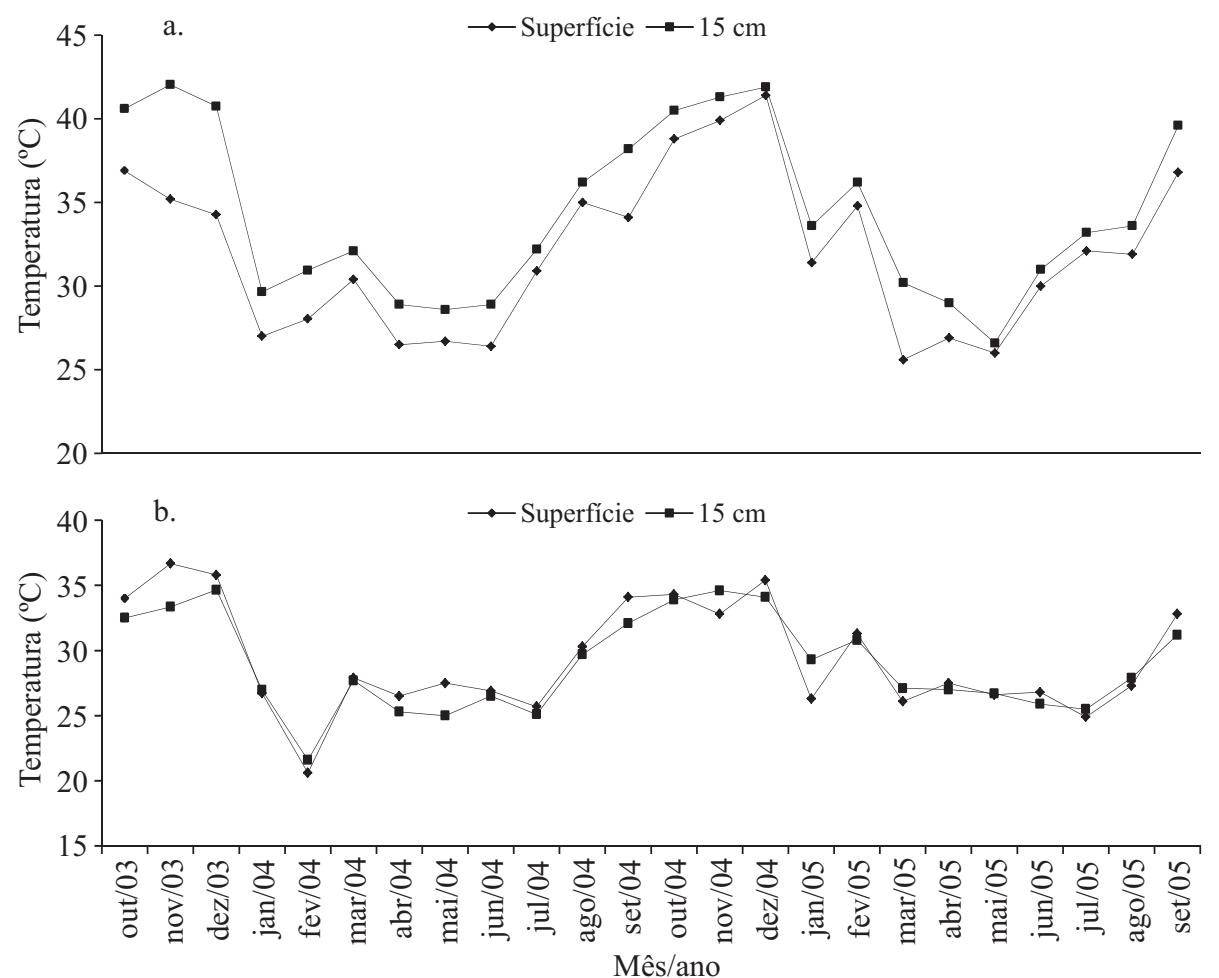

Figura 2 - Flutuação da temperatura do solo na superfície e a 15,0 cm de profundidade nos turnos diurno (a) e noturno (b), durante o período experimental.

Figure 2 - Fluctuation of soil temperature on the surface and $15.0 \mathrm{~cm}$ deep in the day (a) and nocturne shift (b) during the experimental period.

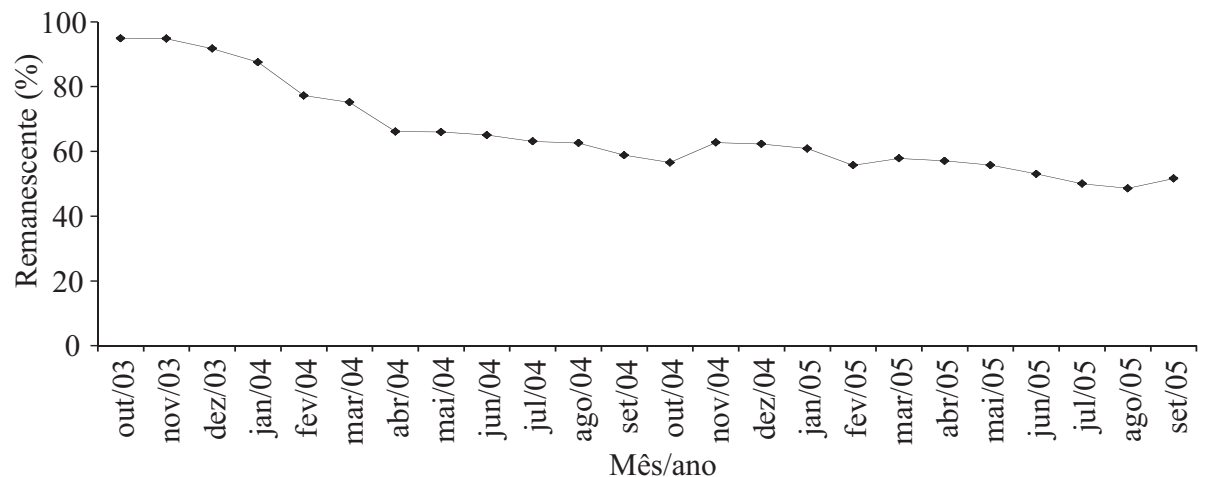

Figura 3 - Serapilheira remanescente (\%) ao longo do processo de decomposição em área de Caatinga, durante as 24 coletas realizadas no período experimental.

Figure 3 - Litterfall remaining (\%) during the decomposition process in an area of Caatinga during the 24 samples collected in the experimental period.

Nos primeiros 12 meses (Período 1), o modelo com melhor ajuste para estimar a perda de massa foi o quadrático: $y=31,61142-1,92128 x+0,06370 x^{2} \quad$ com
$R^{2}=0,915121\left(R_{a j}^{2}=0,911349\right)$. Nesse período, na estação chuvosa (dezembro de 2003 a julho de 2004), ocorreu um aumento da taxa de decomposição, onde o percentual

Cerne, Lavras, v. 19, n. 4, p. 559-565, out./dez. 2013 
remanescente ficou em cerca de $63 \%$. É provável que, nesse período, a serapilheira apresentasse maior concentração de componentes que foram lixiviados, como os extrativos, e substâncias mais simples como açúcares, que são facilmente metabolizados pelos microrganismos. Além disso, é importante ressaltar que a serapilheira contida nas sacolas apresentava ainda grande quantidade de folhas, que são facilmente degradadas, favorecendo a decomposição mais rápida quando as condições climáticas foram favoráveis.

Segundo Torres et al. (2005), o processo de decomposição da liteira é caracterizado por uma taxa de decomposição alta e um padrão invertido em termos de velocidade em cada fase do processo. Ambas as características são fortemente dependentes do clima local e da composição química da liteira.

Como a maioria dos trabalhos sobre decomposição, utilizando a técnica das sacolas de náilon, enfoca apenas o material foliar e o conhecimento desse processo em área de Caatinga é escasso, dificulta as comparações, visto que a serapilheira é composta de frações lábeis e frações estáveis que demoram mais tempo para se decompor.

Verifica-se que, nos meses de novembro e dezembro de 2004 e janeiro de 2005 , ocorreu uma pequena elevação no percentual remanescente, provavelmente decorrente do crescimento de raízes novas para dentro da bolsa e, além disso, o material contido nas bolsas foi acumulando partículas de solo que foram carreadas durante as chuvas e não removidas na limpeza para posterior pesagem.

Nos últimos 12 meses de experimentação (Período 2), a taxa de decomposição manteve-se estável, com degradação gradativa da serapilheira sem pico de perda de massa, como foi observado nos primeiros meses de experimentação. A decomposição nesse período foi linear $(y=22,75685-0,32177 x)$ e $\mathrm{R}^{2}=0,46$, com baixo grau de previsão. Esse comportamento pode ser atribuído à permanência nas sacolas das estruturas residuais com altos teores de lignina, taninos e outros componentes, dificultando a ação dos decompositores, que vão degradando-a de forma mais lenta.
$\mathrm{O}$ valor $\mathrm{k}$ (Tabela 1), calculado pela expressão $C=C_{o} e^{-k t}$, considerando-se separadamente o Período 1 e 2 e, em seguida os 24 meses de exposição, mostrou-se baixo quando comparado com os valores obtidos por Santana (2005), trabalhando em área de Caatinga no Seridó do Rio Grande do Norte, em folhas de catingueira (Caesalpinia pyramidalis) $(\mathrm{k}=0,1195)$, marmeleiro (Croton sonderianus) $(\mathrm{k}=0,0596)$, pereiro (Aspidosperma pyrifolium $)(\mathrm{k}=0,0649)$ e mistura de folhas $(\mathrm{k}=0,0795)$.

A decomposição mais rápida no Período 1 é justificada pela predominância da fração folhas nas sacolas de náilon, resultando em 41,19\% de perda de massa. Com o aumento do tempo de exposição (Período 2), permaneceram nas sacolas as folhas mais recalcitrantes, as partes mais resistentes das folhas que não foram totalmente decompostas como nervuras e pecíolos, e as demais frações como cascas, algumas sementes e galhos finos, tornando o processo mais lento, com apenas $7,18 \%$ de perda de massa, quando comparado com a biomassa final do Período 1.

O tempo médio para decompor $50 \%$ da serapilheira, foi estimado em 434 dias no Período 1, e em 1072 para o Período 2, um aumento de $59,5 \%$ a mais nesse tempo. Essas estimativas foram bem superiores às relatadas por Santana (2005), onde as folhas estudadas pelo citado autor tiveram um tempo de decomposição bem menor.

A respiração edáfica reflete a atividade biológica na mineralização dos resíduos orgânicos. Com o auxílio dos dados apresentados, delineou-se o processo de evolução durante os dois anos de experimentação (Figura 4). Como o resultado da atividade respiratória foi acompanhado durante dois anos, optou-se por agrupar os valores dos meses que se repetiram e não realizar a análise de cada mês individualmente, já que os resultados refletiram a mesma tendência nos meses avaliados ao longo do período experimental. Assim, nos dados abaixo apresentados, ao invés de se ter dados dos 24 meses, têm-se valores da respiração diurna e noturna registrados em 12 meses.

Tabela 1 - Estimativa da biomassa final (\%), equação exponencial ajustada aos valores das amostras após 12 e 24 meses de decomposição em área de Caatinga, constante de decomposição $(\mathrm{k})$ e tempo necessário para decompor 50\% da biomassa (t).

Table 1 - Estimation of final biomass (\%), exponential equation fitted to these values of the samples after 12 and 24 months of decomposition in an area of Caatinga, the decomposition constant $(k)$ and the required time to decompose $50 \%$ of biomass $(t)$.

\begin{tabular}{lccccccc}
\hline Período & Biomassa final (\%) & Equação & $k\left(\right.$ dias $\left.^{-1}\right)$ & $\mathrm{t}_{0,5}(\operatorname{dias})$ & $\mathrm{t}_{0,5}(\mathrm{meses})$ & $\mathrm{t}_{0,5}($ anos $)$ \\
\hline Período 1 & 58,81 & $\mathrm{C}=30,37 \mathrm{e}^{-0,0479}$ & 0,0479 & 434 & 14,37 & 1,19 \\
Período 2 & 51,63 & $\mathrm{C}=18,99 \mathrm{e}^{-0,01194}$ & 0,0194 & 1072 & 35,73 & 2,94 \\
\hline
\end{tabular}

Cerne, Lavras, v. 19, n. 4, p. 559-565, out./dez. 2013 


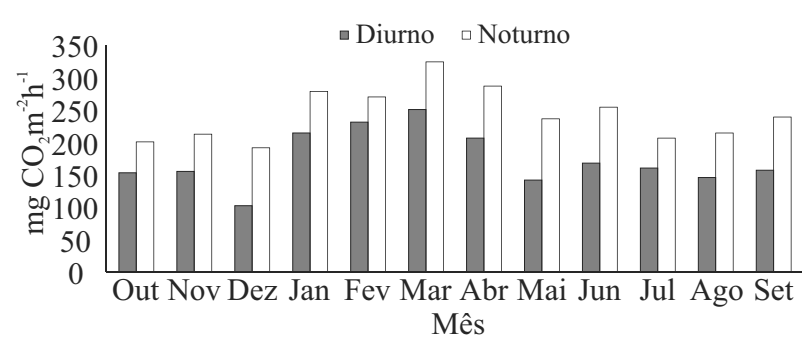

Figura 4 - Média de $\mathrm{CO}_{2}$ liberado em solo sob Caatinga nos turnos diurno e noturno, de outubro de 2003 a setembro de 2005.

Figure 4-Mean $\mathrm{CO}_{2}$ released into the Caatinga soil in day and night shifts, from October 2003 to September 2005.

Ao analisar os resultados, verifica-se que a maior liberação de $\mathrm{CO}_{2}$ ocorreu durante o período noturno, ocorrendo diferença significativa entre os períodos em todas as épocas de amostragem. É provável que, durante a noite, a temperatura do solo nas camadas superficiais, estando mais baixas do que no período diurno (Figura 2), favoreceu a atividade microbiana, resultando em maior liberação de $\mathrm{CO}_{2}$.

Resultados semelhantes foram obtidos por Souto et al. (2002), ao avaliarem a liberação de $\mathrm{CO}_{2}$ em área de Caatinga, após a incorporação de estercos e relataram que, pelas variações térmicas encontradas, o solo funciona como estabilizador térmico.

Segundo Monteiro e Gama-Rodrigues (2004), a atividade microbiana não deve ser analisada de forma isolada e absoluta. $\mathrm{O}$ mais adequado para compreender o funcionamento do sistema solo-serapilheira seria avaliar a inter-relação dos atributos, pois há grande interação e dependência destes na funcionabilidade dos ecossistemas.

\section{CONCLUSÕES}

A decomposição da serapilheira foi maior nos primeiros seis meses de exposição, no início da estação chuvosa, refletindo atividade intensa dos microrganismos

A serapilheira da Caatinga apresentou uma lenta taxa de decomposição, necessitando de mais de 14 meses para que ocorresse $50 \%$ do seu desaparecimento.

A maior atividade microbiana foi verificada no período noturno, favorecida, provavelmente, pela menor temperatura.

\section{AGRADECIMENTOS}

Agradecemos ao Dr. Pierre Landolt, proprietário da Fazenda Tamanduá, pela colaboração e disponibilidade das áreas para a realização da presente pesquisa.

Cerne, Lavras, v. 19, n. 4, p. 559-565, out./dez. 2013

\section{REFERÊNCIAS}

ANDRADE, A. G. Ciclagem de nutrientes e arquitetura radicular de leguminosas arbóreas de interesse para revegetação de solos degradados e estabilização de encostas. 1997. 182 p. Tese (Doutorado em Agronomia) - Universidade Federal Rural do Rio de Janeiro, Seropédica, 1997.

ASSIS JÚNIOR, S. L. de; ZANUNCIO, J. C.; KASUYA, M. C. M.; COUTO, L.; MELIDO, R. C. N. Atividade microbiana do solo em sistemas agroflorestais, monoculturas, mata natural e área desmatada. Revista Árvore, Viçosa, v. 27, n. 1, p. 35 41, fev. 2003.

BAYER, C.; MIELNICZUK, J. Dinâmica e função da matéria orgânica. In: SANTOS, G. A.; CAMARGO, F. A. O. (Ed.). Fundamentos da matéria orgânica do solo: ecossistemas tropicais e subtropicais. Porto Alegre: GENESIS, 1999. p. 9-23.

COSTA, W. A. J. M. de; ATAPATTU, A. M. L. K. Decomposition and nutrient loss from prunings of different contour hedgerow species in tea plantations in the sloping highlands of Sri Lanka. Agroforestry Systems, Heidelberg, v. 51, n. 3, p. 201-211, Mar. 2001.

\section{EMPRESA BRASILEIRA DE PESQUISA}

AGROPECUÁRIA. Manual de métodos de análise de solo. Rio de Janeiro, 1997. 212 p.

GAMA-RODRIGUES, A. C. da; BARROS, N. F.; SANTOS, M. L. Decomposição e liberação de nutrientes do folhedo de espécies florestais nativas em plantios puros e mistos no sudeste da Bahia. Revista Brasileira de Ciência do Solo, Viçosa, v. 27, n. 6, p. 1021-1031, nov./dez. 2003.

GRISI, B. M. Método químico de medição da respiração edáfica: alguns aspectos técnicos. Ciência e Cultura, São Paulo, v. 30, n. 1, p. 82-88, jun./set. 1978.

HOFMAN, J.; DUSEK, L.; KLÁNOVÁ, J.; BEZCHLEBOVÁ, J.; HOLOUBEK, I. Monitoring microbial biomass and respiration in different soils from the Czech Republic: a summary of results. Environment International, New York, v. 30, n. 1, p. 19-30, Mar. 2004.

KOUKOURA, Z.; MAMOLOS, A. P.; KALBURTJ, K. L. Decomposition of dominant plant species litter in a semi-arid grassland. Applied Soil Ecology, Amsterdam, v. 23, n. 1, p. 13-23, May 2003. 
MASON, C. F. Decomposição. São Paulo: EPU, 1980. 63 p.

MONTEIRO, M. T.; GAMA-RODRIGUES, E. F. Carbono, nitrogênio e atividade da biomassa microbiana em diferentes estruturas de serapilheira de uma floresta natural. Revista

Brasileira de Ciência do Solo, Viçosa, v. 28, n. 5, p. 819-826, set./out. 2004.

PARDO, F.; GIL, L.; PARDOS, J. A. Field study of beech (Fagus sylvatica L.) and melojo oak (Quercus pyrenaica Willd) leaf litter decomposition in the centre of the Iberian Peninsula. Plant and Soil, The Hague, v. 191, n. 1, p. 89-100, Apr. 1997.

RODAL, M. J. N.; COSTA, K. C. C.; SILVA, A. C. B. L. Estrutura da vegetação caducifólia espinhosa (Caatinga) de uma área do sertão central de Pernambuco. Hoehnea, São Paulo, v. 35, n. 2, p. 209-217, 2008.

SANTANA, J. A. da S. Estrutura fitossociológica, produção de serapilheira e ciclagem de nutrientes em uma área de Caatinga no Seridó do Rio Grande do Norte. 2005. 184 p. Tese (Doutorado em Agronomia) - Universidade Federal da Paraíba, Areia, 2005.

SAVIN, M. C.; GÖRRES, J. H.; NEHER, D. A.; AMADOR, J. A. Biogeophysical factors influencing soil respiration and mineral nitrogen content in an old field soil. Soil Biology and Biochemistry, Elmsford, v. 33, n. 4/5, p. 429-438, Apr. 2001.
SOUTO, J. S.; ALVES, A. R.; SANTOS, R. V. dos; SOUSA, A. A. de; FREIRE, A. L. de O.; RODRIGUES, C. R. F.; SILVA, G. A. Decomposição de serapilheira de espécies arbóreas, enterrada e aplicada na superfície de um Luvissolo na Paraíba, Brasil. In: REUNIÃO DA SOCIEDADE BRASILEIRA DE CIÊNCIA DO SOLO, 2004, Lages. Anais... Lages: FERTBIO, 2004. 1 CD-ROM.

SOUTO, P. C.; SOUTO, J. S.; SANTOS, R. V. dos; ARAÚJO, G. T. de; MAIA, E. L. Atividade microbiana em função da disposição de estercos no solo. Revista Científica Rural, Bagé, v. 7, n. 2, p. 104-115, dez. 2002.

VANHALA, P. Seasonal variation in the soil respiration rate in coniferous forest soils. Soil Biology and Biochemistry, Oxford, v. 34, n. 9, p. 1375-1379, Sept. 2002.

TAUK, S. M. Biodegradação de resíduos orgânicos no solo. Revista Brasileira de Geociência, São Paulo, v. 20, n. 1/4, p. 299-301, jan./abr. 1990.

TORRES, P. A.; ABRIL, A. B.; BUCHER, E. H. Microbial succession in litter decomposition in the semi-arid Chaco woodland. Soil Biology \& Biochemistry, Oxford, v. 37, n. 1, p. 49-54, Jan. 2005.

WITKAMP, M. Decomposition of leaf litter in relation to environment microflora and microbial respiration. Ecology, Durham, v. 47, n. 1, p. 194-201, 1966.

Recebido: 20 de março de 2011; aceito: 24 de maio de 2013.

Cerne, Lavras, v. 19, n. 4, p. 559-565, out./dez. 2013 
